\title{
Quantitative Analysis of Materials Structures Based on 3D Image Data
}

\author{
Katja Schladitz*, Michael Godehardt*, Christian Kübel** \\ * Department of Image Processing, Fraunhofer Institut für Techno- und Wirtschaftsmathematik \\ ** Institute of Nanotechnology, Karlsruhe Institute of Technology , Germany
}

In this contribution, we demonstrate how to use integral-geometric methods and the concept of a macroscopically homogeneous random closed set in order analyze quantitatively the complex microor nano-structure of modern materials, based on $3 \mathrm{~d}$ image data obtained e.g. by micro computed tomography, FIB tomography or electron-tomography as well as 2d SEM images. Main emphasis is on electron tomography for materials structures and the particular demands on image processing and analysis made by this imaging method.

A basic set of characteristics for objects are the intrinsic volumes - in $2 \mathrm{~d}$ area, perimeter length and Euler number, in $3 \mathrm{~d}$ volume, surface area, the integral of mean curvature and the Euler number. If there is no natural particle, cell or pore structure, whole materials components can be described using the densities of the intrinsic volumes instead. Combining integral and digital geometry yields an efficient algorithm for simultaneous calculation of the intrinsic volumes based on local image information, only. From the intrinsic volumes and the generalized projections connected with them, valuable information e.g. about coarseness, connectivity, or anisotropy of the structure can be gained $[1,2]$. Moreover, local structural information like local orientation or thickness can be gained by methods of mathematical morphologicy.

An indispensible prerequisite for correct quantitative analysis is a correct segmentation of the component or the objects of interest. An extensive simulation study [3] showed that usually used tomographic reconstruction algorithms induce a decline of mean grey value with particle size. That is, in the reconstructed tomographic image, small particles are darker. This effect requires special care when segmenting particles for further analysis and hampers unbiased automatic segmentation, in particular in electron-tomographic images. Nevertheless, a combination of thresholding methods and morphological transforms yields results compatible with a sophisticated partially interactive segmentation that had been suggested in [3]. The opportunities and limitations for automatic segmentation of electron-tomographic images are discussed and illustrated by examples.

\section{References}

[1] Ohser, J., Nagel, W., Schladitz, K., Image Anal. Stereol. 28(2) (2009) 77-92

[2] Ohser, J., Schladitz, K.: 3d Images of Materials Structures - Processing and Analysis. Wiley, Heidelberg, 2009

[3] Kuebel, C., Godehardt, M., Cieslinski, R., Rozeveld, S., Microscopy and Microanalysis 15 (2009) 602-+ 